\title{
Erratum to: How MIC Is Detected and Recognised?
}

\section{Erratum to: \\ Chapter 6 in: R. Javaherdashti, Microbiologically Influenced Corrosion, Engineering Materials and Processes, DOI 10.1007/978-3-319-44306-5_6}

The book was published with incorrect text in Chap. 6 which has been revised now as follows:

In P. 113, Line 340: The paragraph starting with "If we want to compare ..." has been replaced with revised content "If we want to compare the MMM with conventional culture-dependent methods (such as MPN), we can see that while MPN results may not be matched with the risk of MIC, culture-independent methods ( $q$ PCR) are both applicable in more convenience to risk-based inspection and assessments and do show a good correlation with the risk of MIC".

In P. 114, Line 345 in Para 1: The paragraph starting with "Table 6.2" and ending with "these method" has been replaced with revised content "One has to keep this in mind that at the moment all these methods (both culture-based and culture-independent methods) are classified as laboratory methods in the sense that in order to perform them, one has to have both a well-equipped laboratory and trained personnel. This may appear as a disadvantage for those clients who count beans" but one has to observe that the highly precise results that are obtained from MMM tests with regards to MIC risk does compensate the cost spent on these methods."

The updated original online version for this chapter can be found at 10.1007/978-3-319-44306-5_6

R. Javaherdashti ( $\square$ )

Perth, Western Australia, Australia

e-mail: Javaherdashti@yahoo.com

(C) Springer International Publishing Switzerland 2017

E1

R. Javaherdashti, Microbiologically Influenced Corrosion,

Engineering Materials and Processes, DOI 10.1007/978-3-319-44306-5_12 
Figure 6.8 and its legends have been removed and other figures were renumbered accordingly.

Table 6.2 and its legends have been removed. 